\title{
Un camino iniciado y parcialmente recorrido: concepciones y expectativas de los futuros docentes sobre el Máster en Profesor de Educación Secundaria una década después
}

\author{
José María MARTÍNEZ-FERREIRA \\ Diego MIGUEL-REVILLA \\ María SÁNCHEZ-AGUSTÍ
}

Datos de contacto:

José María Martínez-Ferreira Universidad de Valladolid josemaria.martinez.ferreira@uva.es

Diego Miguel-Revilla Universidad de Valladolid diego.miguel.revilla@uva.es

María Sánchez-Agustí Universidad de Valladolid maria.sanchez.agusti@uva.es

Recibido: 23/03/2021 Aceptado: 17/07/2021

\section{RESUMEN}

Más de una década después de la implantación del Máster en Profesor de Educación Secundaria como modelo de formación docente, son muchos los retos que todavía quedan pendientes. Esta investigación presenta un análisis de las concepciones y el imaginario docente de los profesores de Educación Secundaria en formación mediante un examen de las respuestas proporcionadas por 594 estudiantes matriculados en las distintas especialidades del Máster en la Universidad de Valladolid. La información ha sido obtenida a lo largo de cuatro cursos académicos, entre el año 2015 y el año 2019. A través de un análisis de carácter mixto, se examinan las motivaciones, dedicación y perspectivas laborales del futuro profesorado, así como sus expectativas y concepciones sobre el modelo de formación docente. Los resultados dejan patente que un porcentaje significativo de los participantes no muestra un interés por dedicarse prioritariamente a la docencia, y que casi la mitad compagina sus estudios de Máster con trabajos u otros estudios. Igualmente, se identifican diversas competencias que los docentes en formación esperan desarrollar a lo largo del Máster, encontrando contrastes según la tipología de los futuros profesores, y ofreciendo una visión sobre su imaginario docente. Asimismo, se presenta un examen acerca de las percepciones de los estudiantes sobre el propio Máster como modelo formativo y sobre las alternativas existentes, abordando en la discusión final algunos de los retos futuros en la formación del profesorado de Secundaria.

PALABRAS CLAVE: Formación inicial del profesorado; Imaginario docente; Máster en Profesor de Educación Secundaria; Educación Secundaria 


\title{
A road taken and only partially travelled: conceptions and expectations of prospective teachers about the Master Degree in Secondary Education Teaching after a decade
}

\begin{abstract}
A decade after the implementation of the Master's Degree in Secondary Education Teaching as the framework for teacher training, there are many challenges that still remain. This research provides an analysis of the conceptions and of the consciousness as teachers of trainee Secondary Education educators by examining the responses provided by 594 students enrolled in the different areas in the Master's Degree at the University of Valladolid. Information was obtained over four academic years, between 2015 and 2019. Using a mixedmethod design, the motivations, dedication and occupational outlook of trainee teachers were examined, as well as their expectations and conceptions about the teaching training model. Results evidence that a significant percentage of the participants did not show interest in pursuing a career in teaching, and that nearly half of them combine their Master's Degree studies with jobs or other studies. Likewise, a series of competences that pre-service teachers hope to develop in the course of the Master's Degree were identified, while contrasts were found taking into account the typology of trainee teachers, which offered a perspective of their consciousness as educators. An examination of students' perceptions about the Master's Degree as the framework for teacher training, as well as about potential alternatives is also presented. A discussion is offered at the end, covering some of the future challenges in initial teacher training for Secondary Education.
\end{abstract}

KEYWORDS: Initial teacher training; Teacher consciousness; Master's Degree in Secondary Education teaching; Secondary Education 


\section{Introducción}

Formar a los futuros profesores, y además formarlos adecuadamente, son reclamaciones que constantemente se recogen de manera explícita en la legislación, en los medios de comunicación o en los debates en el seno de la sociedad. Pese a ello, es posible detectar multitud de resistencias que frenan potenciales avances, y aunque la inercia suponga una barrera, ésta no debe ocultar los esfuerzos realizados y algún camino ya iniciado y aún no recorrido en la formación del profesorado de Secundaria. Abordar estas problemáticas supone, además, reflexionar sobre lo que se ha denominado imaginario docente, entendiendo éste como el conjunto de ideas y competencias para usar y transformar el conocimiento base en un conocimiento educativo (González-Gallego, 2005), algo que debe analizarse en relación con la tensión entre formación científica y formación profesionalizadora, en el seno de las exigencias y demandas sociales y de las propuestas institucionales para darles respuesta.

Se ha optado por fundamentar la investigación en este concepto frente al más estructurado de "identidad profesional" (Bolívar, 2006), por considerarlo más apropiado para analizar las ideas, expectativas y motivaciones de unos profesores que todavía no lo son y que, por tanto, carecen del proceso de socialización e integración en una "comunidad de práctica" en los centros educativos, circunstancia de crucial importancia en la configuración de la identidad docente. El imaginario docente formaría parte, pues, de las representaciones sociales o conocimiento ordinario de los futuros profesores, que tiene como particularidad la de ser socialmente construido y compartido en el seno del colectivo del que forma parte (profesorado en formación inicial) y que "en los campos profesionales, expresa la manera en que los actores se sitúan con relación a su actividad y a sus compañeros" (Jodelet, 2011, p. 134). Esta representación social del saber escolar se caracteriza por estar desvinculada de su formación inicial y el elevado porcentaje de fracaso escolar en las aulas de Secundaria (Rivero \& Souto, 2019).

El docente de Educación Secundaria tiene que ser profesor de una enseñanza generalista, básica, obligatoria y gratuita para la totalidad de los adolescentes: la ESO. Pero tiene que ser también, y simultáneamente, profesor de una enseñanza selectiva de carácter preuniversitario: el Bachillerato, planteando una dicotomía que exige a los docentes una identidad dual o disociativa de difícil manejo. Al profesor se le demanda que sea especialista en un campo disciplinar, lo que le obliga a desarrollar unos currículos extensos para los que no tiene tiempo y, al mismo tiempo "debe asumir un compromiso personal en la educación de alumnos y alumnas, con crecientes déficits de socialización, convirtiéndose en educador de actitudes y valores, y orientador de sus vidas" (Bolívar, 2004, p. 12).

Una relación compleja de la formación de estos profesionales, y no siempre bien resuelta desde el ámbito institucional, que crea fuertes tensiones y discrepancias en la consideración de la presencia, caudal y sentido de la formación pedagógica y didáctico-disciplinar de los futuros docentes. Esta complejidad queda reflejada en la estructura y organización de la formación inicial del profesorado, con un componente general y otro profesional, que pueden organizarse de diversas formas. 
En el modelo simultáneo, el diseño del título incorpora paralelamente la formación científica correspondiente a las materias a impartir y la formación didáctica y pedagógica del futuro docente, orientada desde el inicio a la formación de un profesional de la educación, como es el caso de Austria, Rumanía o Polonia. Para Esteve (2003) este modelo produce una identidad profesional más adecuada a la realidad, conjugando una formación específica y práctica del futuro docente, que abarca tanto los aspectos académicos y curriculares como los derivados de los nuevos retos que debe de afrontar. Y es que, como apunta el mismo autor, cuando se estudia una disciplina científica con la mentalidad de enseñarla, se van elaborando estrategias didácticas al mismo tiempo que se asimilan los contenidos científicos.

En el modelo consecutivo, adoptado principalmente por los países de la Europa occidental como Francia, Italia o España, primero se adquiere la formación disciplinar, obteniendo el título de Grado correspondiente, para posteriormente abordar la formación didáctico-pedagógica mediante un Máster a través del cual se cualifican para el ejercicio docente en la Educación Secundaria. Desde este modelo, los profesores son formados, primero, como científicos en los distintos campos del saber para, posteriormente, "añadir" una formación, mucho más breve, como profesores. Ello produce, como señala Esteve (2003), que los futuros docentes de secundaria se imaginen a sí mismos más como historiadores o químicos que como profesores de Historia o de Química, generando el problema de la "falta de imaginario docente" (González-Gallego, 2010), caracterizado por la consideración de que son los conocimientos disciplinares los que conforman la cultura académica del profesorado y por ende su identidad profesional (Cuesta et al., 2010).

Otra de las limitaciones del modelo consecutivo, cuando el Máster profesionalizador se circunscribe a un curso académico, es la parquedad de la experiencia práctica en el proceso de formación inicial. Este es el caso español, de ahí que, en nuestro país, desde hace años, se viene hablando de un proyecto, conocido popularmente como MIR educativo, que hasta el momento no ha cristalizado en un proyecto real. En él, el acceso a la formación docente se articularía en un primer año teórico-práctico de nivel de Máster, con un número de plazas vinculado a las necesidades docentes reales, y una segunda fase de dos años de duración, de prácticas remuneradas y tuteladas en un centro educativo (Prats el al., 2019).

\section{Una década de formación del profesorado en el Máster de Secundaria}

La implantación en España del Máster en Profesor de ESO y Bachillerato, Formación Profesional y Enseñanza de Idiomas en el curso 2009/2010, impulsado por la necesidad de adaptar las titulaciones al Espacio Europeo de Educación Superior, supuso, sin duda, un avance en la capacitación profesional del docente de Secundaria (Viñao, 2013), corrigiendo el deplorable deterioro en el que, con el transcurso de los años, se había sumido el proceso de obtención del CAP. El anterior intento de sustitución por unos estudios de mayor calado y consideración, como fue el caso del Curso de Cualificación Pedagógica, no llegó a cuajar más que en contadas universidades como la Universidad Autónoma de Barcelona o la Universidad de Valladolid. Las resistencias a abandonar el imaginario docente tradicional, unido a la 
facilidad de obtener un certificado de capacitación en unas pocas semanas, hubieran sido difíciles de romper sin la ayuda del proceso de Bolonia y el proceso de armonización de titulaciones universitarias (Manso \& Valle, 2013). Desde entonces ha transcurrido algo más de una década y son varios los artículos que han analizado la formación del Profesorado de Secundaria a raíz del desarrollo del Máster como modelo formativo (García et al., 2010).

Uno de los estudios que primero abordó el análisis de las expectativas depositadas por el alumnado en el Máster fue el de Zagalaz et al. (2011), donde se detectó, entre los participantes de la Universidad de Jaén, bajos niveles de valoración en el cumplimiento de las expectativas generadas. Los autores consideraron que ello podía estar condicionado por la comparación con el modelo formativo del CAP, más asequible, breve en tiempo y dedicación, que acababa de desaparecer. En esos años iniciales, el trabajo de Solís et al. (2013) presenta también datos semejantes. Centrados en la especialidad de Ciencias, recabaron información en un grupo de la Universidad Complutense de Madrid y en diversas universidades públicas de Andalucía durante el curso 2010-2011. Estos investigadores encontraron que los docentes en formación, aunque afirmaron haber conseguido una idea general sobre la finalidad y contenidos del área específica, manifestaban unas expectativas no demasiado elevadas en relación con el grado de preparación para impartir clases. Estos resultados han sido refrendados por estudios más recientes, detectando contrastes sobre el cumplimiento de las expectativas en las valoraciones del alumnado y los docentes (Cachón et al., 2015). Investigaciones más recientes, como la llevada a cabo por Rivero y Souto (2019) con el alumnado del Máster de las universidades de Valencia y Zaragoza, indican un alto grado de satisfacción académica con estos estudios.

Otro núcleo de investigaciones ha abordado las estimaciones de los futuros profesores sobre las competencias docentes y su consecución por medio de las asignaturas cursadas en el Máster. Ya en el primer año de su implantación, Buendía et al. (2011) realizaron un estudio en la Universidad de Granada, cuyos participantes asignaron las valoraciones más elevadas a competencias principalmente relacionadas con el aprendizaje y enseñanza de las materias de la especialidad correspondiente, tales como conocer estrategias y técnicas de evaluación, o la elaboración de propuestas educativas, entre otras. Pontes et al. (2013), en un estudio con alumnos de la especialidad de Ciencias del Máster en la Universidad de Córdoba, emplearon una metodología de análisis cualitativo, detectando que estos profesores en formación valoraban principalmente conocimientos profesionales de tipo práctico y el desarrollo de competencias docentes relacionadas con la metodología de enseñanza, la utilización de recursos y la gestión del aula. En sentido parecido, Martínez et al. (2019), en su trabajo sobre alumnos de la especialidad de Geografía e Historia, señalan que éstos principalmente aspiran a adquirir conocimientos didácticos de cara a implementar la docencia y una base teórico-práctica útil en su inicio como docentes.

Asimismo, Serrano y Pontes (2015), en su investigación con 353 estudiantes del conjunto de especialidades del Máster en la Universidad de Córdoba, indicaron, como competencias más destacadas por el alumnado, la adquisición de estrategias para estimular el esfuerzo de los estudiantes y promover su capacidad de aprendizaje 
autónomo y colaborativo, así como la planificación, desarrollo y evaluación de los procesos educativos con estrategias adecuadas al nivel y desarrollo competencial de sus futuros alumnos. Al respecto, Sola et al. (2020) realizaron un estudio con el alumnado del Máster en la Universidad de Granada, en el curso 2019-20, identificando una mayor valoración de los aspectos referidos a estimular la motivación por el aprendizaje o fomentar el espíritu crítico y emprendedor. En cambio, curiosamente, los estudiantes manifestaron menor interés hacia las competencias relativas a los conocimientos didácticos respectivos o a la planificación, desarrollo y evaluación de los procesos de enseñanza-aprendizaje, entre otros.

Un tercer objeto de estudio sobre el Máster de Secundaria se ha focalizado en las prácticas docentes. Sin duda, un elemento crucial de la formación del profesorado de Secundaria lo constituye el Practicum, en la medida que supone la inmersión en la labor docente (Madalena Calvo et al., 2020; Sans, 2013). Igualmente, incide en la conformación de la identidad profesional que, en la mayor parte de las ocasiones, se empieza a configurar a partir del desarrollo de las prácticas (Peinado \& Abril, 2016). En este sentido, González-Gallego (2010) señala la importancia de la elección de los centros y tutores para proporcionar a los alumnos del Máster una buena experiencia que incentive una docencia positiva e innovadora. Y es que, en ocasiones, el alumnado encuentra obstáculos para realizar innovaciones pedagógicas en el desarrollo de las prácticas docentes (Valdés \& Bolivar, 2014). Por otra parte, también se ha detectado una complacencia espontánea con su actuación en el aula y una autosuficiencia en la observación y análisis de las personas mentoras, aunque luego no se refleja en datos empíricos en los informes (Madalena Calvo et al., 2020).

De forma significativa, Serrano y Pontes (2017), en su investigación centrada en las diferencias entre expectativas y logros en las competencias del Practicum, detectaron un contraste entre la valoración atribuida a la importancia de las competencias que se desarrollan durante la fase de prácticas, y el nivel de desarrollo que los estudiantes consideran haber alcanzado al finalizar las mismas. Por otro lado, Torrego (2015), en su trabajo sobre la valoración del Practicum por parte del alumnado que cursó el Máster en la Universidad de Valladolid en el curso 2011-12, identificó que un 89 por ciento de los encuestados consideraron el Practicum como la fase más importante de su formación profesionalizadora. En esta línea, Manso y Martín (2014) también detectaron una alta valoración y un alto nivel de acuerdo respecto a la propuesta de una mayor extensión del tiempo dedicado a las prácticas. Estos trabajos reseñados coinciden en reflejar una alta expectativa y valoración hacia la fase práctica del Máster de Secundaria, independientemente del contexto geográfico y temporal.

\section{Objetivos y preguntas de investigación}

Teniendo en cuenta los aspectos descritos con anterioridad, se tomó la decisión de realizar un estudio en el ámbito de la Universidad de Valladolid, con la finalidad de recabar información sobre el proceso de formación de los estudiantes que cursan el Máster en Profesor de ESO y Bachillerato, Formación Profesional y Enseñanza de Idiomas, y poder contrastar los datos obtenidos en otras investigaciones y contextos. 
Partimos de la idea de que la profesión docente es un recurso de última hora para muchos de los estudiantes del Máster, de ahí la ausencia de un imaginario docente cuando ingresan en el Máster y la insuficiente respuesta institucional para construirlo con un curso de un año de duración.

Para ello, se ha utilizado una selección representativa del alumnado que cursa diferentes especialidades de dicho Máster, a lo largo de cuatro cursos académicos. Ha interesado averiguar, especialmente, los motivos que llevan al alumnado a matricularse en el Máster, su dedicación y perspectivas de futuro. Además, esta investigación también se interesa por conocer la manera en que perciben estos estudios y las expectativas generadas en torno a ellos al comenzar el curso, así como las concepciones del futuro profesorado acerca del modelo formativo. A través de esta información, se pretende obtener una visión del imaginario docente de los futuros profesores que en los próximos años se incorporarán a las aulas de Secundaria en sus diversas disciplinas. Por tanto, la investigación parte de las siguientes preguntas de investigación:

(1) ¿Cuáles son los motivos por los que los alumnos que acceden a los estudios del Máster en Profesor de Educación Secundaria han decidido cursarlo? ¿Cuál es su grado de dedicación y sus perspectivas profesionales de cara al futuro?

(2) ¿Cuáles son las expectativas sobre el Máster con las que los docentes en formación comienzan dichos estudios?

(3) ¿Cuáles son sus concepciones sobre el modelo de formación docente para Educación Secundaria?

\section{Método}

La presente investigación empírica asume un diseño de carácter mixto con una implementación concurrente, tratando de suplementar información cuantitativa con aportaciones puntuales de carácter cualitativo capaces de ayudar en la interpretación (Greene, 2007). El estudio hace uso, en todo caso, de un enfoque predominantemente cuantitativo de carácter descriptivo (Bisquerra, 2004), con la finalidad de proporcionar una visión de conjunto de la situación y las ideas de los participantes, atendiendo a escalas de valoración y diversas categorías preestablecidas. Al asumir un diseño de carácter CUAN-cual (Hernández Sampieri et al., 2014), estos datos cuantitativos han sido, en ocasiones, suplementados con expresiones espontáneas de tipo cualitativo.

\section{Contexto y participantes}

Para la puesta en marcha de este estudio ha sido posible contar con un total de 594 estudiantes matriculados en el Máster en Profesor de Educación Secundaria en la Universidad de Valladolid, en cuatro cursos académicos, entre el año 2015 y el año 2019. El 50 por ciento de dichos participantes tenía una edad de menos de 25 años al cumplimentar el cuestionario, mientras que más de un 26 por ciento se encontraba entre 25 y 29 años, y cerca de un 23 por ciento tenía más de 30 años. En total, pudo contarse con las respuestas de 350 mujeres y 240 hombres (cuatro personas no 
indicaron este aspecto). Con la finalidad de lograr la máxima representatividad posible en la selección de los participantes, la investigación ha tratado de obtener información del mayor número posible de estudiantes matriculados, abarcando alumnado de todas las especialidades ofertadas. El contraste numérico entre especialidades es un reflejo de la cantidad de estudiantes matriculados anualmente en cada una de ellas y los numerus clausus establecidos para cada una de ellas, cifra muy dispar. Desde este punto de vista, el muestreo puede ser descrito como no probabilístico e intencional, así como de carácter típico (Wellington, 2015). Al respecto, es posible observar la distribución de los participantes según las diferentes especialidades ofertadas en la Tabla 1.

\section{Tabla 1}

Distribución de los profesores en formación según la especialidad cursada

\begin{tabular}{lrr}
\hline Especialidad cursada en el Máster & $\mathrm{n}$ & $\%$ \\
\hline Lenguas Extranjeras & 144 & 24.2 \\
Geografía e Historia & 88 & 14.8 \\
Lengua castellana y literatura & 54 & 9.1 \\
Tecnología e informática & 47 & 7.9 \\
Matemáticas & 43 & 7.2 \\
Biología y Geología & 42 & 7.1 \\
Física y Química & 40 & 6.7 \\
Economía & 31 & 5.2 \\
Música & 22 & 3.7 \\
Orientación educativa & 22 & 3.7 \\
Formación y Orientación Laboral & 16 & 2.7 \\
Latín y Griego & 16 & 2.7 \\
Filosofía & 14 & 2.4 \\
Tecnología agraria, alimentaria y forestal & 9 & 1.5 \\
Intervención sociocomunitaria & 6 & 1.0 \\
\hline Total & 594 & 100 \\
\hline
\end{tabular}

\section{Obtención y procesamiento de la información}

La información fue recopilada en cada uno de los cursos académicos durante el primer mes tras el comienzo del Máster. Para ello, se hizo uso de un cuestionario semiestructurado en papel, que fue cumplimentado en persona por los estudiantes gracias a la colaboración del profesorado encargado de la docencia. Los participantes ofrecieron su consentimiento informado con la finalidad de que los datos proporcionados pudieran ser usados en la presente investigación. El instrumento, de carácter anónimo, estuvo conformado por tres preguntas semi-cerradas de elección múltiple en la que los estudiantes pudieron clarificar sus respuestas (y en las que 
pudieron añadir de manera espontánea respuestas abiertas), por un apartado completamente abierto, y por siete ítems de valoración a través de una escala de tipo Likert (utilizando una escala del 1 al 10, según el grado de acuerdo con cada afirmación). La realización de una prueba de fiabilidad en aquellos ítems centrados en las expectativas didácticas sobre el Máster de los participantes indicó un grado elevado de consistencia interna $(\alpha=.77)$, al superar el umbral requerido de $\alpha=.70$. Los datos cuantitativos fueron trasladados y procesados mediante el software SPSS, lo que permitió llevar a cabo un análisis de carácter descriptivo. Por otro lado, la información cualitativa fue transcrita y codificada haciendo uso de categorías emergentes utilizando el software ATLAS.ti (Figura 1).

\section{Figura 1}

Vista de red de las categorías cualitativas analizadas

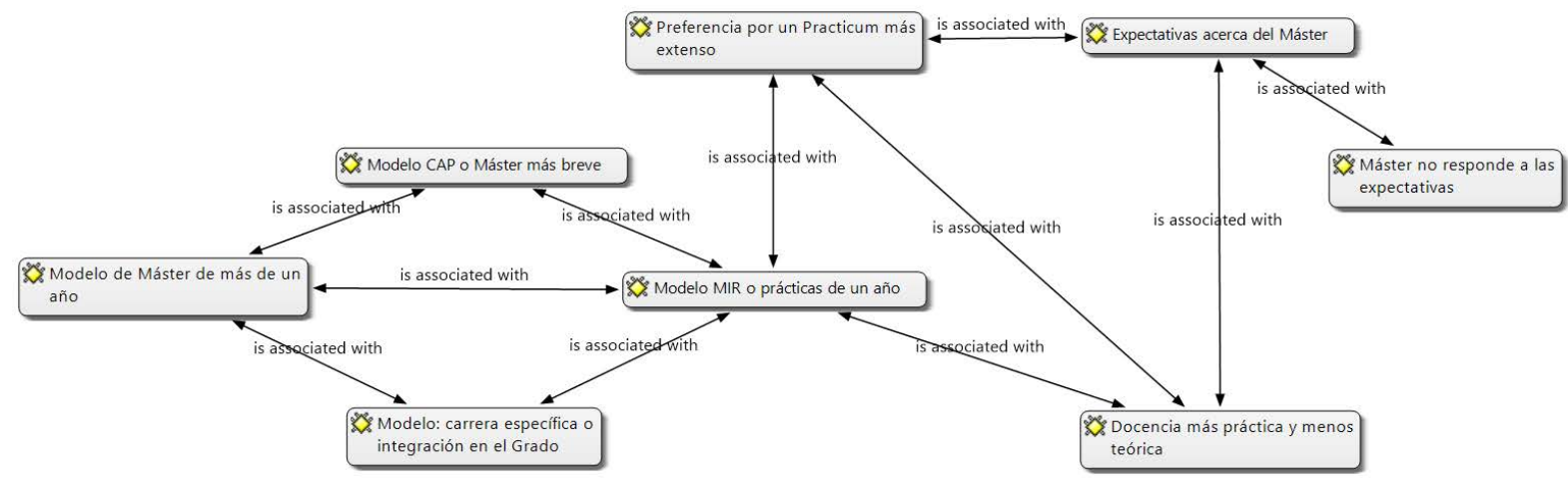

Se parte de una categoría relacionada con las expectativas generadas por el Máster, de la que derivan ideas ligadas con el modelo de formación docente y con la extensión y rol de las prácticas en los centros. Así, esta categoría inicial se relaciona estrechamente con otra, focalizada en agrupar aquellas respuestas en las que los participantes inciden en aquellos factores por los que el Máster no responde a sus expectativas previas. A la vez, la categoría inicial también se relaciona con otras, en las que los docentes en formación expresan su preferencia por un modelo menos teórico, o por un Practicum más extenso. Esta última idea se liga, asimismo, a los diversos modelos de formación docente a los que hacen referencia los estudiantes: las cuatro categorías se interrelacionan, reflejando diferentes concepciones sobre la manera en la que debería estructurarse la formación del profesorado de Secundaria.

\section{Resultados}

\section{Motivaciones y perspectivas laborales del futuro profesorado}

En relación con el primero de los objetivos del estudio, se decidió llevar a cabo un análisis utilizando la información proporcionada por los participantes sobre sus 
ideas. Es por ello, por lo que se examinó tanto su valoración sobre las razones por las que decidieron cursar el Máster como su dedicación y perspectivas de futuro.

En primer lugar, atendiendo a los motivos por los que se cursa el Máster, es necesario señalar que, como se refleja en la Figura 2, menos de la mitad de los profesores en formación encuestados declaran que querían dedicarse a la docencia desde el momento en que comenzaron a cursar sus estudios de Grado o Licenciatura (un 46.6 por ciento de los 594 participantes) y de ahí el deseo de matricularse en este Máster profesionalizante. Por el contrario, un 15 por ciento indica, explícitamente y de manera espontánea, que tomó la decisión tras finalizar sus estudios de Grado, al considerar, solamente entonces, esta salida profesional como algo factible, mientras que otro 2.4 por ciento cita la obligatoriedad del Máster como motivo para cursarlo. Junto a ellos, se usan otros argumentos espontáneos de carácter educativo: un 4.2 por ciento de los participantes cita su interés por conocer o profundizar en el mundo de la docencia y otro 1.9 por ciento expresa el atractivo de conocer el funcionamiento de la etapa de Secundaria.

Por otro lado, el resto de participantes dejan de lado los argumentos de carácter educativo y se centran, en cambio, en aspectos ligados al mundo del trabajo (27 por ciento de los 594 participantes). En este caso, como respuesta más frecuente (22.1 por ciento del total), los futuros docentes argumentan que cursan estos estudios por si no encuentran otro trabajo y tienen que dedicarse a la docencia. A este porcentaje se suman otras argumentaciones escritas de manera espontánea por los participantes: un 4.2 por ciento que afirman tener trabajo, pero buscan un cambio, así como un 0.7 por ciento que indica haber escogido el Máster al encontrarse en paro. A todos ellos se añade un 3.1 por ciento adicional que no responde o no específica sus respuestas.

\section{Figura 2}

Motivos de los participantes para cursar el Máster

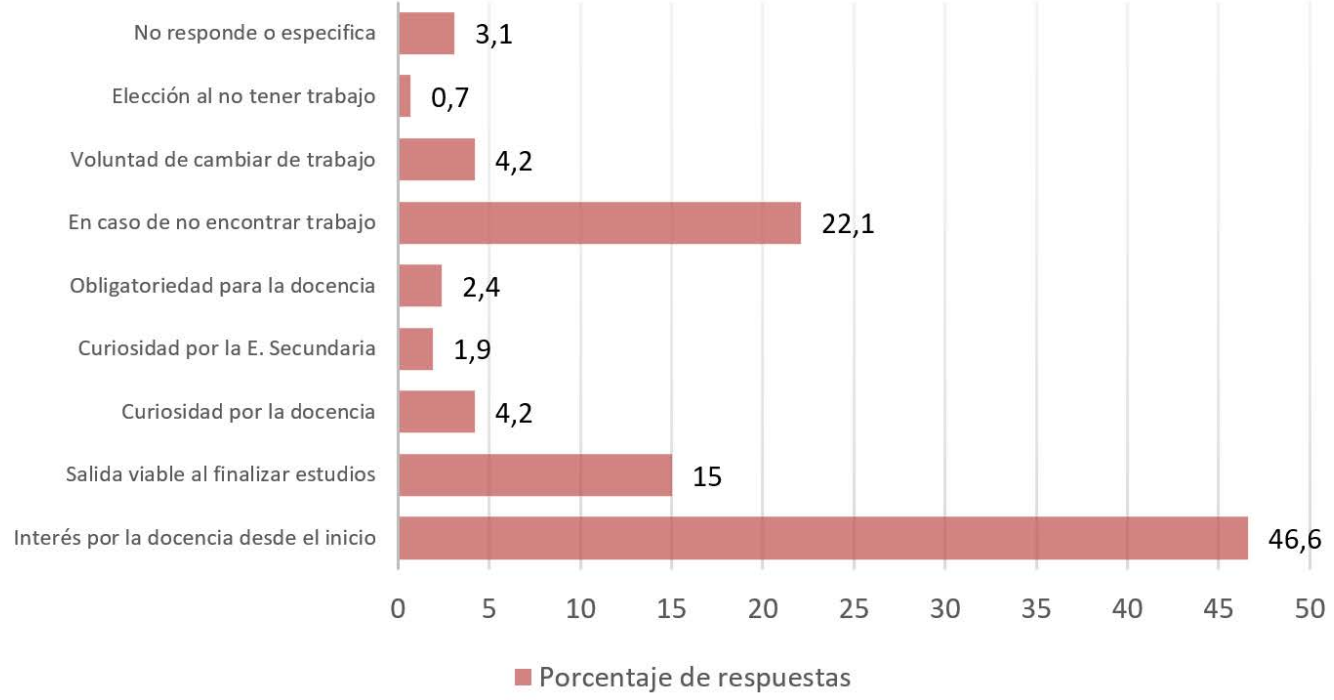


En segundo lugar, y poniendo el foco en esta ocasión en la dedicación de los profesores en formación durante su periodo en el Máster, se detecta un panorama complejo. Solamente un 49.3 por ciento de los 594 participantes afirma cursar estos estudios con una dedicación exclusiva. Por otro lado, un 9.4 por ciento de los futuros docentes indica que compatibiliza su dedicación al Máster con otros estudios. Dentro de este grupo, la respuesta más habitual (4.2 por ciento) es la compaginación con otros estudios de postgrado, incluyendo estudios de Doctorado, seguido por otros estudios de Grado (1.2 por ciento). Otro 7.9 por ciento de los participantes encuestados indica que cursa estudios de idiomas de forma paralela y un 4 por ciento no especifica el tipo de estudios que está realizando.

Por otro lado, la cifra de docentes en formación que compagina sus estudios con un trabajo es elevada: un 31.1 por ciento. Tras realizar un examen de las respuestas proporcionadas, un 11.4 por ciento del total de los futuros docentes trabaja en ocupaciones relacionadas con el mundo de la educación no reglada. Un 7.6 por ciento se emplea, en cambio, en ocupaciones derivadas de su formación académica, pero de carácter no educativo. Finalmente, un 12.1 por ciento afirma tener ocupaciones no relacionadas con ninguno de estos aspectos (trabajo en la hostelería o comercio, entre otras ocupaciones). El 2.2 por ciento restante no responde a esta pregunta.

También se interrogó a los docentes en formación sobre sus perspectivas de futuro una vez finalizado el Máster. En esta ocasión, la respuesta mayoritaria es la preparación de oposiciones, opción seleccionada por un 56.6 por ciento de los 594 participantes (ver Figura 3). Un 24.7 por ciento de los mismos señala en sus respuestas la expectativa o preferencia de trabajar, en cambio, en un centro educativo concertado o privado. Por otro lado, y fuera ya del marco docente, un 5.9 por ciento de los futuros profesores indica en sus respuestas que tienen la intención de seguir estudiando una vez que acaben el año de formación en el Máster, en esta ocasión, generalmente, en estudios ligados a su ámbito disciplinar. Un 4.7 por ciento adicional se decanta, asimismo, por ponerse a trabajar, aunque en esferas no ligadas al mundo educativo. El 8.1 por ciento restante señala otras opciones, mayoritariamente ligadas a viajar al extranjero (para la mejora del idioma o para la búsqueda de oportunidades), pero también se encuentran referencias a la preparación de oposiciones ligadas a otras profesiones o a continuar con la ocupación laboral vigente, entre otros aspectos secundarios). Por tanto, cabe destacar que en torno a una quinta parte de los participantes no parece tener claro dedicarse a la docencia en un futuro próximo.

Cruzando los diferentes datos, es posible observar que aquellos profesores en formación que afirman que querían dedicarse a la docencia desde el inicio tienen un 52.3 por ciento de dedicación exclusiva (frente a la media general de 49.3 por ciento), y un 67.5 por ciento quiere preparar oposiciones en el futuro (frente a la media global de un 56.6 por ciento), indicando un mayor nivel de focalización en sus perspectivas ligadas al mundo laboral en el ámbito educativo. 


\section{Figura 3}

Perspectivas de futuro de los participantes tras finalizar el Máster

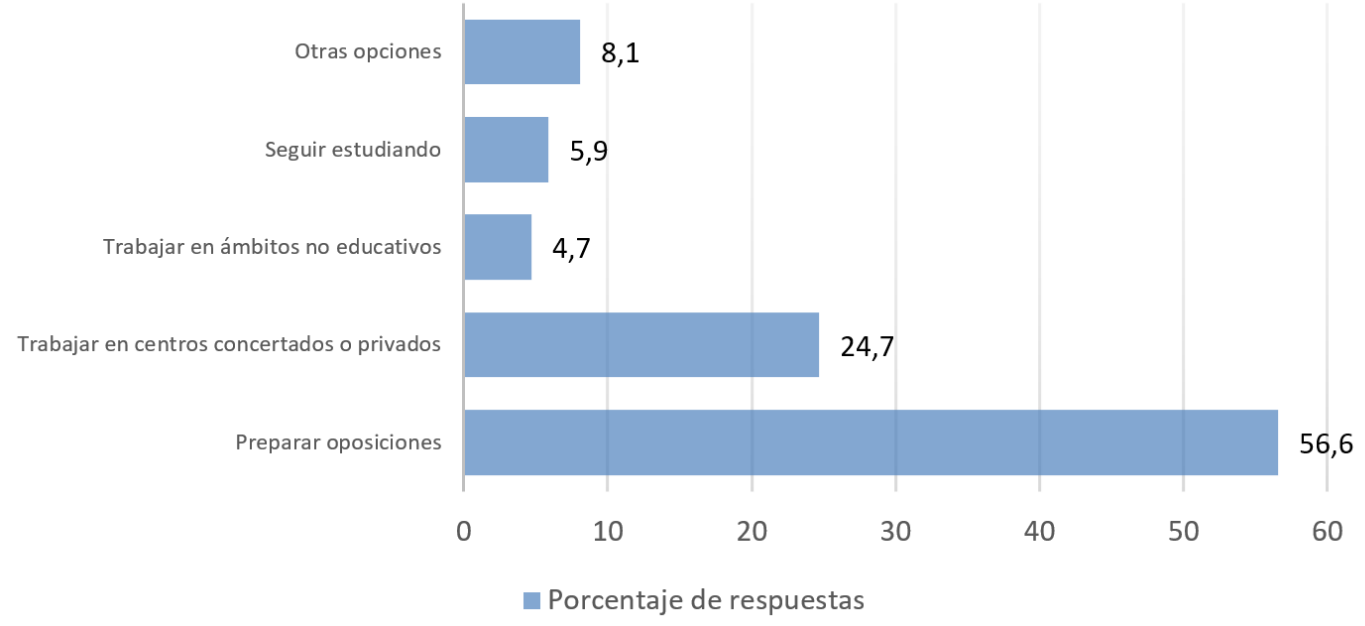

\section{Expectativas del profesorado en formación sobre el Máster}

Una vez examinada la situación de la que parten los docentes en formación al comenzar el Máster, tanto en lo relacionado a sus motivaciones, dedicación y perspectivas de futuro, se analizan a continuación sus expectativas sobre estos estudios. En primer lugar, como puede observarse en la Tabla 2, los participantes muestran un grado de acuerdo muy alto ante tres afirmaciones concretas, ya que esperan del Máster que les ofrezca información sobre el funcionamiento de los centros educativos, contenidos propios del ámbito de la organización escolar $(M=$ 8.21), sobre las características de los alumnos que pueden encontrarse en el futuro, aspectos generalmente abordados desde la psicología $(M=8.11)$ o sobre conocimientos y destrezas para afrontar con éxito la docencia de la materia correspondiente, contenidos abordados desde las didácticas específicas $(M=8.40)$. Todos estos aspectos, ligados con una visión de carácter práctico y focalizada en adquirir competencias para el desarrollo profesional docente, son valorados de forma similar entre los diferentes grupos, aunque con pequeños contrastes.

Así, es posible detectar valoraciones algo más elevadas, aunque no sustanciales, entre aquellos docentes que afirmaron haber estado interesados por la docencia desde el inicio de sus estudios $(M=8.30, M=8.21$ y $M=8.47$, respectivamente) en contraposición al resto $(M=8.21, M=8.03$ y $M=8.35$, respectivamente). Igualmente, aquellos docentes en formación que compaginan sus estudios de Máster con otro tipo de estudios muestran valoraciones más bajas en los tres ítems $(M=7.70, M=7.68$ y $M$ = 7.90, respectivamente) frente, tanto a quienes están estudiando el Máster con dedicación exclusiva, como a los participantes que lo compaginan con un trabajo, que son, precisamente, los que parten de expectativas más elevadas $(M=8.28, M=8.32 \mathrm{y}$ $M=8.48$, respectivamente).

148 ISSN 0213-8646 | E-ISSN 2530-3791 • Revista Interuniversitaria de Formación delProfesorado, 96 (35.2) (2021), 137-158 


\section{Tabla 2}

Expectativas de los participantes sobre el Máster

$M \quad$ Des. Est.

\begin{tabular}{lll}
\hline Incrementar conocimientos de la materia a impartir & 6.43 & 2.79 \\
Información sobre el funcionamiento de los centros educativos & 8.21 & 1.72 \\
Conocer las características del alumnado & 8.11 & 1.85 \\
Que aporte conocimientos y destrezas para afrontar con éxito la & 8.40 & 1.89 \\
docencia & 5.08 & 2.49
\end{tabular}

Ya poseo los conocimientos, únicamente necesito practicar en un centro

Nota. Número de respuestas entre $N=586$ y $N=592$ dependiendo del ítem.

En segundo lugar, los futuros docentes ofrecen únicamente un moderado grado de acuerdo con la expectativa de que estos estudios les ayudarán a incrementar sus conocimientos sobre la materia a impartir $(M=6.43)$. Esta visión, por la cual el Máster debería centrarse en abordar contenidos de carácter disciplinar evidencia una brecha entre los profesores en formación dependiendo de la especialidad cursada. Así, el grado de acuerdo con este aspecto es mucho más elevado entre los participantes cursando especialidades de humanidades o ciencias sociales $(M=6.81)$ frente a los que cursan especialidades técnicas o no sociales $(M=5.57)$. Es igualmente llamativo que aquellos docentes en formación que indican haber mostrado un mayor interés por la docencia desde el inicio también tienen unas expectativas más elevadas al respecto $(M=6.83)$ que el resto de sus compañeros $(M=6.08)$ en relación a este ítem.

En tercer lugar, preguntados por su grado de acuerdo sobre si consideran que ya poseen los conocimientos y sólo requieren cursar las prácticas en los centros, muestran una mayor división de opiniones $(M=5.08)$. Este nivel de acuerdo, el más bajo entre todos los ítems, no muestra variaciones de relevancia entre los diferentes tipos de docentes en formación, ya se atienda a su interés por la docencia o especialidad. En todo caso, el grupo que considera que su nivel de preparación es el más elevado es el de aquellos participantes que compaginan el Máster con otros estudios $(M=5.41)$, mientras que quienes muestran mayores dudas son, precisamente, aquellos profesores en formación con dedicación exclusiva durante sus estudios $(M=4.92)$.

Estas respuestas han sido complementadas con las opiniones que escribieron varios de los participantes al cumplimentar los cuestionarios. En esta ocasión, de las 144 respuestas recogidas, sólo es posible encontrar 28 menciones que, de manera espontánea, hacen referencia a sus expectativas sobre el Máster.

Entre las diversas ideas, aquellos estudiantes que expresan su opinión muestran una cierta unanimidad en el hecho de que el Máster debería centrarse en aspectos didácticos o pedagógicos, pues, en palabras de un encuestado, es necesario "que las clases estén orientadas a 'cómo enseñar X materia', no a los contenidos de la materia en 
sí (vistos durante el Grado)" (I.19.GH.4). Esta idea se repite: "creo que es muy importante la formación psicológica y didáctica pedagógica" (I.16.BI.4); o "El 'saber enseñar/educar' antes que los contenidos" (I.16.MU.5).

Por otro lado, las reflexiones de los futuros docentes también ofrecen una ventana ante la que asomarse para conocer aspectos que describen su imaginario docente. Este es el caso de un participante, quien afirma que "debería ser un máster más práctico y menos teórico", y, por tanto, "enfocado a aprender ciertos 'trucos' para poder ser profesor" (I.19.BI.2). La concepción de la docencia como una mera práctica basada en trucos o despojada de una base teórica es, de hecho, un aspecto que debería tenerse en cuenta, ya que puede llegar a ser prevalente en ciertos docentes en formación. A su vez, es posible encontrar visiones en las que, pese a que el foco principal es de carácter educativo, los aspectos puramente más prácticos o utilitarios pueden generar una mayor preocupación entre los futuros docentes. Este es el caso de un encuestado que indica que "a la hora de enfrentarnos a una clase, a ganar confianza y perder el miedo a los alumnos" (I.16.FIL.4), así como otro compañero suyo, que cree que el Máster debería enfocarse a "proporcionarnos las herramientas necesarias para manejar de forma eficiente a los alumnos; es decir, saber actuar en situaciones difíciles, de bullying, etc." (I.19.BI.3).

Más allá de estas afirmaciones que ofrecen información sobre la concepción y expectativas sobre el Máster, entre las opiniones de los participantes se encuentran siete encuestados que citan su decepción con el mismo. En estos casos no se citan razones específicas salvo en una ocasión, en la que se señala que "me hubiera gustado un panorama teórico más breve y que realmente me prepare a ser profesora", señalando a continuación que querría aprender cómo "actuar adecuadamente ante los adolescentes que serán mis alumnos... en fin, que [el Máster] sea mucho más profesionalizante" (I.19.LE.15). Precisamente, una mayor incidencia en aspectos prácticos es recalcada por 22 estudiantes, generalmente señalando la importancia de conocer cómo gestionar aspectos del mundo real frente a información de carácter teórico, a lo que se unen 10 menciones explícitas a la duración del periodo de prácticas, ya que para estos encuestados "el Practicum debería ser más largo, pues es la aplicación de esos conocimientos adquiridos" (I.17.MAT.4).

\section{Concepciones en torno al modelo de formación docente en Secundaria}

Finalmente, y con la finalidad de examinar aspectos complementarios capaces de proporcionar información adicional sobre el imaginario docente del futuro profesorado, se tomó la decisión de examinar sus concepciones sobre el modelo de formación para ser profesor de Educación Secundaria. Para ello, se solicitó a los participantes que valoraran, utilizando una escala entre 1 y 10 , su grado de acuerdo con el requerimiento de un año de formación didáctica y pedagógica tras haber cursado un grado universitario, así como con otra posibilidad alternativa en la que se requeriría cursar un Grado universitario destinado exclusivamente a la formación docente en Educación Secundaria, donde se compaginarían los aspectos disciplinares con los profesionalizantes. Tras el análisis de los resultados, los futuros docentes se inclinan por el modelo de formación a través del Máster $(M=6.40)$, en comparación 
con el modelo alternativo propuesto que, por otro lado, no registra un rechazo elevado $(M=4.44)$.

El modelo formativo consecutivo es valorado mucho más entre los 293 participantes que se dedican exclusivamente a sus estudios de Máster $(M=6.57)$ y los 182 que los compaginan con un trabajo $(M=6.36)$, frente a los que 102 participantes que compaginan el Máster con otros estudios $(M=5.77)$. En cambio, los resultados respecto a la alternativa (modelo concurrente) no muestran diferencias relevantes. Llama la atención que los docentes en formación en especialidades de Humanidades y Ciencias Sociales valoran siempre más positivamente ambas opciones $(M=6.50$ y $M=$ 4.63, respectivamente), frente a las respuestas encontradas por los futuros docentes en especialidades técnicas o no sociales $(M=6.17$ y $M=4.02$, respectivamente).

Por último, de nuevo se han examinado las respuestas abiertas que, de forma espontánea, fueron escritas por los futuros docentes. Aunque solamente un número muy bajo de estudiantes abordan específicamente este asunto, es posible observar diferentes percepciones acerca de lo que debería ser el modelo formativo para Secundaria. Como muestra, seis participantes señalan su preferencia por un modelo en el que el Máster se alargara durante dos años, con el objetivo, en palabras de un participante, de evitar "tanta carga de trabajo en poco tiempo" (I.15.LE.12). Uno de los docentes en formación se decanta por esta opción argumentando que sería adecuado "un año la formación teórica y un año la práctica" (I.15.LE.27). Esta última idea se relaciona con un aspecto señalado por tres participantes adicionales, quienes señalan que "las prácticas tendrían que ser como el MIR de los médicos, ya que no todos los futuros profesores tienen vocación” (I.17.LE.10), en palabras de uno de ellos.

Ocho participantes indican espontáneamente, en cambio, que se decantarían por la "creación de un grado exclusivo para la docencia o inclusión de especialidad en los grados existentes" (I.17.LI.19), según escribe uno de ellos. Entre los argumentos se encuentran visiones muy claras acerca de lo que debería ser la preparación de los docentes, como puede observarse en apreciaciones como la siguiente: "la formación del profesor debe ser constante: ni en un año ni en un grado. Un año nos puede habilitar para dar clase, pero no puede acabar ahí nuestra formación pedagógica" (I.16.GH.15). Ahora bien, también es posible encontrar tres docentes en formación adicionales que se decantan por modelos previamente vigentes. En palabras de uno de ellos, "creo que el formato anterior (CAP) era mejor. Me parece muchísimo tiempo, dado que es dedicar otro año entero a la universidad" (I.16.LE.12), lo que ofrece una visión muy distinta.

\section{Discusión y conclusiones}

Una vez expuestos los resultados obtenidos a partir de la información recabada a lo largo de cuatro cursos académicos, a continuación se presenta una interpretación de los mismos, de cara a poder abordar los objetivos y preguntas de investigación planteados inicialmente.

En primer lugar, esta investigación perseguía identificar los motivos por los que el profesorado de Educación Secundaria en formación accede a los estudios de Máster, así como su grado de dedicación y perspectivas profesionales. El análisis de las razones por las que se cursa el Máster ha permitido destacar que menos de la mitad 
del profesorado de Secundaria en formación (46.6 por ciento) manifiesta una vocación docente como profesor al iniciar sus estudios universitarios. Por otro lado, un porcentaje muy significativo de los futuros profesores que cursa el Máster afirma querer dedicarse a la docencia únicamente si no encuentra trabajo en otro campo ligado a su formación inicial del Grado. Análisis similares sobre los motivos por los que se cursa el Máster, como los realizados por Solís et al. (2013) o por Martínez et al. (2019), señalan, en consonancia, que en muchas ocasiones los estudiantes cursan estos estudios al ser un requisito, o porque "nunca se sabe", y no tanto por motivación propia o percepción sobre su utilidad. El hecho de que, por tanto, muchos de los estudiantes no estén matriculados en el Máster por vocación, o que la docencia no sea su interés principal, puede provocar que su imaginario docente no sea el adecuado.

El grado de dedicación de los estudiantes del Máster también ha sido abordado en la presente investigación, lo que ha llevado a detectar que un porcentaje muy significativo de ellos compaginan sus estudios con un trabajo (31.1 por ciento). Estos datos resultan muy parejos a los detectados por Zagalaz et al. (2011) en la Universidad de Jaén, con cifras también cercanas al 30 por ciento. Rivero y Souto (2019) mostraron que en la Universidad de Zaragoza, un tercio del alumnado compatibilizaba sus estudios con una actividad laboral, una cifra también muy similar. En el caso de la Universidad de Valladolid, es necesario añadir a este número que un 17.3 por ciento de los matriculados compagina el Máster con otros estudios. Estos datos reflejan una situación que afecta a casi la mitad de los profesores en formación, lo que puede explicar ciertas tensiones y malestar entre los estudiantes por la sobrecarga de trabajo.

De igual modo, la investigación ha permitido identificar que un 81.3 por ciento de los encuestados afirma querer trabajar como profesor de Secundaria en el futuro. Esta cifra se aproxima a las de otros estudios similares, como el llevado a cabo en la Universidad de Jaén, en el que el 89.1 por ciento de los estudiantes aspiraban a dedicarse a la docencia (Zagalaz et al., 2011). Aun así, resulta llamativo que un porcentaje destacable se decante por otras opciones profesionales no ligadas al mundo educativo. En el caso de la Universidad de Valladolid, casi uno de cada cinco matriculados no tiene perspectivas de dedicarse al mundo de la docencia en el futuro y, a pesar de ello, optan por cursar un Máster profesionalizante.

Este estudio también marcó como su segundo objetivo analizar las expectativas con las que el profesorado en formación comienza el Máster, y ha detectado que, en el imaginario docente de los participantes, adquirir los conocimientos y destrezas necesarios para desarrollar exitosamente la docencia de la materia correspondiente ocupa un lugar primordial. Junto a esto, se valoró como necesario dominar aspectos organizativos y conocer las características del alumnado. Estas consideraciones coinciden por las expuestas por Buendía et al. (2011), así como por Martínez et al. (2019) y Sola et al. (2020), donde también se identificó un alto grado de valoración a competencias similares.

La expectativa de incrementar los conocimientos de la materia a impartir fue muy inferior en este caso, lo que contrasta con los resultados obtenidos por Serrano y Pontes (2015), aunque en el presente estudio se han detectado contrastes entre determinadas especialidades. En el caso de Geografía e Historia, Física y Química, o 
Biología y Geología, donde se valora de manera más alta esta necesidad, las diferencias pueden deberse a que el alumnado proviene de Grados de formación disciplinar que abordan una única materia, provocando carencias formativas respecto a los contenidos que deberán impartir. Éste sería el caso de la especialidad en Geografía e Historia, en la que, como indican Rivero y Souto, los docentes en formación "no han cursado una carrera que les proporcione conocimientos avanzados de las tres disciplinas" (2019, p. 48), lo que requiere completar estos déficits en su formación disciplinar inicial. Esta idea también se corrobora desde la posición del alumnado, tal y como señala Sans (2013).

Por otro lado, algunos de los aspectos detectados en las respuestas abiertas también corroboran la importancia que muchos otorgan a las competencias docentes percibidas con un carácter más práctico (gestión del aula, manejo de situaciones conflictivas, etc.), en línea con el estudio cualitativo de Pontes, Serrano y Poyato (2013). Precisamente, esta idea ya fue recalcada con anterioridad por Suárez (2010), quien manifestó que el alumnado del Máster persigue una formación funcional como objetivo prioritario teniendo en mente el proceso de acceso a la profesión docente a través de las oposiciones. Como apunta este autor, "el Máster no es el único responsable en la formación de futuros profesores” (Suárez, 2010, p. 9), ya que persiste un sistema selectivo diseñado por la Administración que puede influir en las expectativas de los docentes en formación. Igualmente, es importante destacar que, de acuerdo con los resultados obtenidos, los docentes en formación son bastante conscientes de sus carencias profesionalizadoras: pese a haber cursado sus estudios de Grado, reconocen mayoritariamente que todavía no cuentan con las competencias necesarias para el ejercicio de la docencia en Secundaria. Esto se refleja en sus expectativas, puesto que consideran que estas carencias podrán subsanarse a través del Máster.

Esta circunstancia presenta, además, una relación con las concepciones del alumnado sobre el modelo de formación docente, algo que ha sido abordado en el último apartado de este estudio. Atendiendo a las valoraciones de los participantes, éstos parecen haber asumido como adecuado el hecho de tener que cursar un año de formación didáctica y pedagógica tras sus estudios de Grado. Parecen haberse superado las reticencias encontradas en los primeros años de implantación del Máster, en los que los profesores en formación atribuían una excesiva duración, costes económicos y dedicación temporal a estos estudios de postgrado, teniendo en mente el antiguo modelo del CAP (Cachón et al., 2015). Tras el examen de las respuestas abiertas encontradas en el presente estudio, el recuerdo del CAP parece haberse diluido.

Es significativo que tampoco manifiesten un alto rechazo ante la propuesta de cursar un Grado destinado a la formación docente en Educación Secundaria, siendo más receptivos los estudiantes del ámbito de Humanidades y Ciencias Sociales, donde la enseñanza constituye una de las pocas salidas laborales. Asimismo, a través de las respuestas abiertas se apuntaron otras opciones, como una mayor dedicación de tiempo a las prácticas, en concordancia con lo reflejado en las investigaciones de Manso et al. (2014) o de Torrego (2015), la ampliación del Máster a dos años de duración, o la inclusión de asignaturas orientadas a la docencia en los estudios de 
Grado, coincidiendo con lo expuesto por Suárez (2010) y Sans (2013), desde su perspectiva como ex-alumnos del Máster. Incluso se encontraron varias citas espontáneas que toman el modelo MIR como referencia.

\section{Implicaciones y futuras líneas de trabajo}

Como ha podido observarse, el actual modelo parece haber conseguido superar algunas de las reticencias iniciales de los futuros docentes de Secundaria. En todo caso, son muchos los retos que han podido ser detectados, y que inciden tanto en aspectos organizativos como académicos de los profesores en formación inicial que cursan el Máster. Es el caso del hecho de que, pese a requerir dedicación exclusiva (dada la carga horaria y de inmersión en contenidos para los alumnos que lo cursan), se constata que en torno a la mitad de los matriculados en el Máster lo compagina con otras actividades.

Junto a esto, teniendo en cuenta que casi la mitad de los encuestados manifiesta que quería dedicarse a la docencia cuando inició sus estudios de Grado, cabría realizar una reflexión sobre la pertinencia de incluir un itinerario formativo orientado a la docencia en Secundaria, en las titulaciones de procedencia de algunas especialidades, o un Grado específico para la enseñanza en esta etapa. La consideración, además, de modelos formativos alternativos es uno de los grandes debates que están sobre la mesa (Prats el al., 2019) y pueden ser informados por análisis de la situación actual, como el aquí presentado.

Este estudio asumió este reto, y aunque únicamente se ha analizado al alumnado matriculado en la Universidad de Valladolid y, por tanto, existen limitaciones en relación a la muestra, el hecho de que los datos se hayan recabado a lo largo de cuatro cursos académicos, y que se haya contado con un número muy elevado de docentes en formación, ofrecen fortaleza a la investigación. Este estudio puede constituir, junto con otros trabajos mencionados, una base sobre la que poder extraer conclusiones una década después de la implantación del Máster de Secundaría. Un camino iniciado y parcialmente recorrido que no puede tener éxito, como indica Tiana (2013) "sin haber abordado otros aspectos como el acceso a la carrera docente, los incentivos para el acceso a la misma, y los medios para su mejora" (p. 56). Precisamente, esto marca también futuras líneas de investigación, en las que poder profundizar sobre la valoración y percepción del desarrollo competencial por parte del profesorado en formación que cursa el Máster, teniendo en cuenta los retos a los que se enfrenta el desarrollo de la identidad profesional de los futuros docentes en un mundo cada vez más complejo.

\section{Agradecimientos}

Esta investigación ha sido realizada en la Facultad de Educación y Trabajo Social de la Universidad de Valladolid. Para ello se ha contado con el permiso y apoyo de la Institución, así como con el conocimiento y consentimiento de los estudiantes que han participado, todos ellos mayores de edad. Los autores agradecen a la Universidad y al alumnado del Máster la colaboración prestada, sin la cual no hubiera sido posible la investigación. 


\section{Conflicto de intereses}

Los autores declaran no tener ningún conflicto de intereses.

\section{Contribuciones de los autores}

Conceptualización, J.M.; metodología, J.M., D.M. y M.S.; recopilación de datos, J.M y M.S., análisis de datos J.M., D.M. y M.S., redacción, revisión y edición, J.M., D.M. y M.S.

\section{Referencias}

Bisquerra, R. (Ed.). (2004). Metodología de la investigación educativa. Editorial La Muralla.

Bolívar, A. (2004). La educación secundaria obligatoria en España. En la búsqueda de una inestable identidad. Reice. Revista electrónica Iberoamericana sobre Calidad,

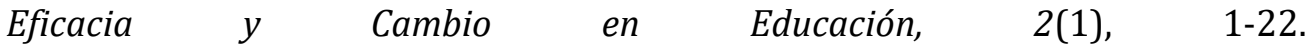
https://revistas.uam.es/reice/article/view/5534

Bolívar, A. (2006). La identidad profesional del profesorado de secundaria: crisis y reconstrucción. Ediciones Aljibe.

Buendía, L., Berrocal, E., Olmedo, E. M., Pegalajar, M., Ruiz, M.A., \& Tomé M. (2011). Valoración por parte del alumnado de las competencias que se pretenden conseguir con el Máster universitario de Profesorado de Educación Secundaria Obligatoria, Bachillerato, Formación Profesional y Enseñanza de Idiomas. Bordón, 63(3), 57-74. https://recyt.fecyt.es/index.php/BORDON/article/view/29055

Cachón, J., López, I., Romero, S., Zagalaz, M. L. \& González, C. (2015). Opinión de docentes y estudiantes del máster de secundaria sobre las aportaciones de este a la formación del profesorado, la calidad docente y los intereses personales. Magister, 27, 1-10. https://doi.org/10.1016/j.magis.2015.03.001

Cuesta, R., Mainer, J., \& Mateos, J. (2010). Más allá de lo obvio. Cuadernos de Pedagogía, 404, 88-91.

Esteve, J. M. (2003). La tercera revolución educativa: la educación en la sociedad del conocimiento. Paidós.

García, F., Solís, E. \& Porlán, R. (2010). El Máster en Sevilla: frustraciones y oportunidades. Cuadernos de Pedagogía, 404, 85-87.

González-Gallego, I. (2005). Una información invertebrada: la de ser profesor (consideraciones sobre la formación docente). En MEC, El profesorado y los retos del sistema educativo actual (pp. 205-260). Secretaria General Técnica MEC.

González-Gallego, I. (2010). Concepto, normativa y propuesta de aplicación para geografía e historia. En I. González-Gallego (Coord.), El profesorado y los retos del sistema educativo actual (pp. 27-73). Graó.

Greene, J. C. (2007). Mixed Methods in Social Inquiry. Jossey-Bass.

Hernández Sampieri, R., Fernández Collado, C., \& Baptista Lucio, P. (2014). Metodología de la investigación (6ª ed.). McGraw-Hill.

Jodelet, D. (2011). Aportes del enfoque de las representaciones sociales al campo de la educación. Espacios en Blanco. Revista de Educación, 21, 133-154. 
Madalena Calvo, J. I., Pinheiro dos Santos, M. F., Rodríguez Pizzinato, L. A., \& Souto González, X. M. (2020). La valoración del Practicum en la formación inicial: un estudio de casos internacional en el área de Geografía e Historia. Revista Interuniversitaria de Formación del Profesorado, 95(34.3), 97-118. https://doi.org/10.47553/rifop.v34i3.81004

Manso, J. \& Martín, E. (2014). Valoración del Máster de Formación de Profesorado de Educación Secundaria: estudio de casos en dos universidades. Revista de Educación, 364, 145-169. https://doi.org/10.4438/1988-592X-RE-2014-364258

Manso, J., \& Valle, J. (2013). La formación inicial del profesorado de secundaria en la Unión Europea. Revista Española de Educación Comparada, 22, 165-184. https://doi.org/10.5944/reec.22.2013.9328

Martínez, R., López, A. B., \& González, I. (2019). Valoración de las expectativas de formación en el Máster de Profesorado de Enseñanza Secundaria en la especialidad de Geografía e Historia. En Hortas, M. J., Dias, A., \& De-Alba, N. (Coord.), Enseñar y aprender didáctica de las Ciencias Sociales: la formación del profesorado desde una perspectiva sociocrítica. AUPDCS - ESE/PL.

Peinado, M., \& Abril, A. M. (2016). El Máster en Profesorado de Secundaria desde dentro: expectativas y realidades del Prácticum. Didáctica de las Ciencias Experimentales y Sociales, 30, 5-22. https://doi.org/10.7203/dces.30.6811

Pontes, A., Serrano, R. \& Poyato, F. (2013). Concepciones y motivaciones sobre el desarrollo profesional docente en la formación inicial del profesorado de educación secundaria. Revista Eureka sobre Enseñanza y Divulgación de las Ciencias, 10 , 533-551. https://revistas.uca.es/index.php/eureka/article/view/2807

Prats, E., Marín, A. \& Álvarez, G. (2019). Elementos para el debate en la iniciación profesional docente en España. En J. M. Valle \& G. Álvarez (Ed.), La iniciación profesional docente: Marcos supranacionales y estudios comparados (pp. 204220). UAM.

Rivero, P. \& Souto, X. (2019). El máster de profesorado de educación secundaria. Una visión desde la especialidad de geografía e historia. Íber Didáctica de las Ciencias Sociales, Geografía e Historia, 97, 41-50.

Sans, R. (2013). Experiencia y valoración acerca del Máster de Secundaria. Íber Didáctica de las Ciencias Sociales, Geografía e Historia, 73, 65-71.

Serrano, R. \& Pontes, A. (2015). Expectativas ante la formación inicial entre el alumnado del Máster de Profesorado de Enseñanza Secundaria. Revista de Investigación Educativa, 33(2), 489-505. https://doi.org/10.6018/rie.33.2.203471

Sola, J. M., Marin Marin, J. A., Alonso Garcia, S., \& Gomez Garcia, G. (2020). Análisis de percepciones del estudiantado del Máster de Secundaria respecto a las competencias profesionales del docente. Revista Electrónica Interuniversitaria de Formación del Profesorado, 23(2), 81-93. https://doi.org/10.6018/reifop.418601

Solís R. E., Martín del Pozo, R., Rivero G. A., \& Porlán A. R. (2013). Expectativas y concepciones de los estudiantes del MAES en la especialidad de Ciencias. 
Revista Eureka sobre Enseñanza y Divulgación de las Ciencias, 10, 496-513. https://revistas.uca.es/index.php/eureka/article/view/2805

Suárez, M. A. (2010). Enseñanza de la Historia: viejos problemas y necesidad de un cambio. Reflexión de un alumno del Máster de Profesorado de Secundaria, CLIO. History and History teaching, 36.

Tiana (2013). Los cambios recientes en la formación inicial del profesorado en España: una reforma incompleta. Revista Española de Educación Comparada, 22, 39-58.

Torrego, A. (2015). El prácticum del máster de secundaria desde dentro. Valoración del alumnado de la Universidad de Valladolid. Íber Didáctica de las Ciencias Sociales, Geografía $\quad e \quad$ Historia, $80, \quad$ 1-6. https://doi.org/10.5944/reec.22.2013.9322

Valdés, R., \& Bolivar, A. (2014). La experiencia española de Formación del Profesorado: El Máster en Educación Secundaria. Ensino em Re-vista,21(1), 159-173. http://www.seer.ufu.br/index.php/emrevista/article/view/25059

Viñao, A. (2013). Modelos de formación inicial del profesorado de Educación Secundaria en España (Siglos XIX-XXI). Revista Española de Educación Comparada, 22, 19-37. https://doi.org/10.5944/reec.22.2013.9321

Wellington, J. (2015). Educational Research: Contemporary Issues and Practical Approaches (2 ${ }^{\mathrm{a}}$ ed.). Bloomsbury.

Zagalaz, M. L., Molero, D., Campoy, T. J., \& Cachón, J. (2011). Las expectativas depositadas en el máster de Educación Secundaria por los futuros docentes durante su formación inicial. Revista de Investigación en Educación, 9(2), 19-34. http://reined.webs.uvigo.es/index.php/reined/article/view/112 
